\title{
Well-posedness and Attractors for a Memory-type Thermoelastic Timoshenko Beam Acting on Shear Force
}

\author{
Soh Edwin Mukiawa* and Cyril Dennis Enyi
}

\begin{abstract}
In this paper, we study the large time behavior of a thermoelastic Timoshenko system with memory effects where the thermoelastic coupling is acting on shear force instead of the bending moment. We establish the existence of finite-dimensional global attractor and an exponential attractor.
\end{abstract}

\section{Introduction}

In this work, we consider a thermoelastic Timoshenko system with memory effects, where the thermoelastic coupling is acting on shear force instead of the bending moment. Precisely, we consider

$$
\begin{cases}\rho_{1} u_{t t}-k\left(u_{x}+v\right)_{x}+\gamma \theta_{x}=0, & x \in(0,1), t>0, \\ \rho_{2} v_{t t}-b v_{x x}+k\left(u_{x}+v\right)-\gamma \theta & \\ \quad+\int_{0}^{+\infty} h(s) v_{x x}(x, t-s) d s+g(v)=f(x), & x \in(0,1), t>0 \\ \rho_{3} \theta_{t}-\beta \theta_{x x}+\gamma\left(u_{x}+v\right)_{t}=0, & x \in(0,1), t>0\end{cases}
$$

with initial data

$$
\begin{cases}u(x, 0)=u_{0}(x), v(x, 0)=v_{0}(x), \theta(x, 0)=\theta_{0}(x), & x \in(0,1), \\ u_{t}(x, 0)=u_{1}(x), v_{t}(x, 0)=v_{1}(x), & x \in(0,1)\end{cases}
$$

and boundary conditions

$$
\left\{\begin{array}{l}
u_{x}(0, t)=v(0, t)=\theta(0, t)=0, \quad t \geq 0 \\
u_{x}(1, t)=v(1, t)=\theta(1, t)=0, \quad t \geq 0
\end{array}\right.
$$

where $u=u(x, t)$ is the transverse displacement, $v=v(x, t)$ is the rotation angle and $\theta=\theta(x, t)$ is the difference temperature. The physical coefficients $\rho_{1}, \rho_{2}, k, \gamma, \beta$ and $b$ are positive constants, $h, g$ and $f$ are given functions to be specified later. There are

Received September 18, 2019; Accepted November 21, 2019.

Communicated by Cheng-Hsiung Hsu.

2010 Mathematics Subject Classification. 35B35, 35B40, 37L05, 37L30.

Key words and phrases. global attractor, exponential attractor, Timoshenko system, thermoelasticity.

*Corresponding author. 
quite a number of results that exist in the literature concerning thermoelastic Timoshenko beam problems. However, a great number of those results are related to the existence, polynomial and exponential stability as well as general stability. For these types of system, see for example [1, 2, 10, 11, 13, 14] and the references therein. For $g=f=0$ and finite memory, system (1.1) has been studied by many authors and various types of stability results have been established. Messaoudi and Fareh [16, 17] considered

$$
\begin{cases}\rho_{1} u_{t t}-k\left(u_{x}+v\right)_{x}+\gamma \theta_{x}=0, & x \in(0,1), t>0 \\ \rho_{2} v_{t t}-b v_{x x}+k\left(u_{x}+v\right)-\gamma \theta+\int_{0}^{t} h(s) v_{x x}(x, t-s) d s=0, & x \in(0,1), t>0 \\ \rho_{3} \theta_{t}-\beta \theta_{x x}+\gamma\left(u_{x}+v\right)_{t}=0, & x \in(0,1), t>0\end{cases}
$$

with Dirichlet boundary conditions and proved a general stability result which depends on the speeds of wave propagation. Apalara 3] studied (1.4) with Neumann-DirichletDirichlet boundary conditions and established a general stability result without imposing any condition on the speed of propagation. The result in 3 shows that the boundary conditions neutralized the effect of the speed. For more existence and stability results related to system (1.4) we refer the reader to $1,4,6,7,12,14,15,18,21$ and references therein. Feng and Yang 9 considered nonlinear Timoshenko beam with a time-delay term and proved the existence of global attractors and exponential attractors. To the best of our knowledge, there are very few results concerning long-time dynamics of thermoelastic Timoshenko beam system in the literature. See for instance the work of Fatori et al. [8]. The main goal in this paper is to study the long-time dynamics of the thermoelastic Timoshenko beam system (1.1)-1.3. Our expected outcomes are as follows:

(i) Under suitable assumptions on the relaxation function $h$ and the nonlinear function $g$, we shall establish the existence of global unique weak solution in appropriate spaces.

(ii) By using the asymptotic smoothness and the stabilizability inequality, we prove the existence of a finite dimensional global attractor.

(iii) The existence of an exponential attractor will be also established.

We should mention that, by imposing a Neumann-Dirichlet-Dirichlet boundary conditions (1.3), we are able to establish our results without any additional condition on the speed of wave propagation, this in itself is quite interesting. In addition, the results of this paper were obtained using a method similar to those used in the study of viscoelastic problems with memory. The remaining part of this work is organized as follows: In Section 2 , we recall some basic tools and assumptions on the relaxation and nonlinear functions $g$ and $h$ respectively. In Section 3 , we establish the well-posedness for system $(1.1)-(1.3)$. In 
Section 4 , we show the existence of global attractor for system (1.1)-(1.3). In Section 5 . we show that the global attractor has a finite fractal dimension. Finally, in Section 6, we establish the existence of an exponential attractor for system (1.1)- 1.3.

Throughout this work, we denote the inner product and norm in $L^{2}(0,1)$ by $(\cdot, \cdot)$ and $\|\cdot\|$ respectively. Also, the letter $C$ or $C_{i}, i=1,2,3, \ldots$, are positive generic constants that may change from one line to another, or perhaps within same line.

\section{Problem setting and preliminaries}

In this section, we recall some useful materials and conditions. For this, we assume that the relaxation function $h$ admits the assumptions:

(A1) The function $h:[0,+\infty) \rightarrow(0,+\infty)$ is an absolutely continuous function satisfying

$$
h(0)>0, \quad b-\int_{0}^{\infty} h(s) d s=b_{0}>0
$$

and there exists a positive constant $\xi$ such that for almost every $y \in \mathbb{R}^{+}$,

$$
h^{\prime}(y)+\xi h(y) \leq 0 .
$$

(A2) We assume $g \in \mathcal{C}^{1}(\mathbb{R})$ and the function $G(s)=\int_{0}^{s} g(\tau) d \tau$ satisfies for some constants $C_{1}, C_{2}>0$

$$
\liminf _{|s| \rightarrow+\infty} \frac{G(s)}{s^{2}} \geq 0, \quad \liminf _{|s| \rightarrow+\infty} \frac{s g(s)-C_{1} G(s)}{s^{2}} \geq 0, \quad g^{\prime}(s) \geq-C_{2}
$$

It's easy to deduce from 2.2 that for any $\rho>0$, there exist constants $C_{\rho}, C_{\rho}^{\prime}>0$ such that

$$
\begin{gathered}
G(s)+\rho s^{2} \geq-C_{\rho}, \\
s g(s)-C_{1} G(s)+\rho s^{2} \geq-C_{\rho}^{\prime}, \quad \forall s \in \mathbb{R} .
\end{gathered}
$$

For instance, the function $g(s)=s|s|^{\gamma}, 0 \leq \gamma<+\infty$, satisfies (2.2).

Denote

$$
m(\phi)=\int_{0}^{1} \phi(x) d x \text { and } \bar{\phi}=\phi-m(\phi) .
$$

Integrating equation 1.1$)_{1}$ over $(0,1)$ and making use of the boundary conditions 1.2 , we obtain

$$
\frac{d^{2}}{d t^{2}} \int_{0}^{1} u(x, t) d x=0, \quad \text { i.e., } \quad m\left(u_{t t}\right)=0 .
$$


We solve (2.5), taking into consideration the initial data of $u$ in $(1.2)$, we get

$$
m\left(u_{t}\right)=m\left(u_{1}\right) \quad \text { and } \quad m(u)=t m\left(u_{1}\right)+m\left(u_{0}\right)
$$

Let

$$
\eta^{t}(x, s)=v(x, t)-v(x, t-s), \quad t, s \geq 0 .
$$

For simplicity of notations, we will sometimes suppress the superscript $t$ of $\eta^{t}$ and write $\eta$ instead of $\eta^{t}$. Therefore, we have

$$
\eta_{t}(x, s)+\eta_{s}(x, s)-v_{t}(x, t)=0, \quad t, s \geq 0 .
$$

Therefore, using (2.6) and replacing $u$ by $\bar{u}$ in (1.1), we have that $(\bar{u}, v, \theta, \eta)$ satisfies

$$
\begin{cases}\rho_{1} u_{t t}-k\left(u_{x}+v\right)_{x}+\gamma \theta_{x}=0, & x \in(0,1), t>0, \\ \rho_{2} v_{t t}-b_{0} v_{x x}+k\left(u_{x}+v\right)-\gamma \theta & \\ -\int_{0}^{+\infty} h(s) \eta_{x x}(x, s) d s+g(v)=f(x), & x \in(0,1), t>0, \\ \rho_{3} \theta_{t}-\beta \theta_{x x}+\gamma\left(u_{x}+v\right)_{t}=0, & x \in(0,1), t>0, \\ \eta_{t}+\eta_{s}-v_{t}=0, & x \in(0,1), t, s>0\end{cases}
$$

with initial data

$$
\begin{cases}\bar{u}(x, 0)=\bar{u}_{0}(x), v(x, 0)=v_{0}(x), \theta(x, 0)=\theta_{0}(x), & x \in[0,1], \\ \bar{u}_{t}(x, 0)=\bar{u}_{1}(x), v_{t}(x, 0)=v_{1}(x), & x \in[0,1], \\ \eta(x, 0)=0, \eta^{0}(x, s)=v_{0}(x)-v(x,-s), & x \in[0,1]\end{cases}
$$

and boundary conditions

$$
\left\{\begin{array}{l}
u_{x}(0, t)=v(0, t)=\theta(0, t)=\eta(0, s)=0, \quad t, s \geq 0 \\
u_{x}(1, t)=v(1, t)=\theta(1, t)=\eta(1, s)=0, \quad t, s \geq 0
\end{array}\right.
$$

We know that $m(\bar{u})=0$, thus we have the Poincaré inequality

$$
\|\bar{\phi}\|^{2} \leq \lambda_{0}\left\|\phi_{x}\right\|^{2} \quad \text { for any } \phi \in H^{1}(0,1) \text {. }
$$

In the rest of this work, $\lambda_{1}$ denotes the Poincaré constant, i.e.,

$$
\|\phi\| \leq \sqrt{\lambda_{1}}\left\|\phi_{x}\right\| \quad \text { for any } \phi \in H_{0}^{1}(0,1)
$$

Set

$$
U=(\bar{u}, \varphi, v, \psi, \theta, \eta)^{T}
$$


Then, system (2.7)-2.9) transforms into the Cauchy problem

$$
U_{t}+A U=F(U), \quad U(x, 0)=U_{0}(x),
$$

where

$$
U_{0}=\left(\bar{u}_{0}, \bar{u}_{1}, v_{0}, v_{1}, \theta_{0}, \eta^{0}\right)^{T}, \quad F(U)=\left(0,0,0,-\frac{1}{\rho_{2}} g(v)+\frac{1}{\rho_{2}} f(x), 0,0\right)^{T},
$$

and the linear operator $A$ is given by

$$
A U=\left(\begin{array}{c}
-\varphi \\
-\frac{k}{\rho_{1}}\left(u_{x}+v\right)_{x}+\frac{\gamma}{\rho_{1}} \theta_{x} \\
-\psi \\
-\frac{\bar{b}}{\rho_{2}} v_{x x}+\frac{k}{\rho_{2}}\left(u_{x}+v\right)-\frac{\gamma}{\rho_{2}} \theta-\frac{1}{\rho_{2}} \int_{0}^{+\infty} h(s) \eta_{x x}(x, s) d s \\
-\frac{\beta}{\rho_{3}} \theta_{x x}+\frac{\gamma}{\rho_{3}}\left(\varphi_{x}+\psi\right) \\
\eta_{s}-\psi
\end{array}\right)
$$

We consider the following space

$$
H_{*}^{1}(0,1)=\left\{w \in H^{1}(0,1) \mid w_{x}(0)=w_{x}(1)=0\right\},
$$

and set

$$
\mathcal{H}=H_{*}^{1}(0,1) \times L^{2}(0,1) \times H_{0}^{1}(0,1) \times L^{2}(0,1) \times L^{2}(0,1) \times \mathcal{M},
$$

where $\mathcal{M}=L_{h}^{2}\left(\mathbb{R}^{+}, H_{0}^{1}(0,1)\right)$ is defined by

$$
L_{h}^{2}\left(\mathbb{R}^{+}, H_{0}^{1}(0,1)\right)=\left\{w:\left.\mathbb{R}^{+} \rightarrow H_{0}^{1}(0,1)\left|\int_{0}^{1} \int_{0}^{\infty} h(s)\right| w_{x}(x, s)\right|^{2} d s d x<+\infty\right\}
$$

endowed with the inner product

$$
\langle w, z\rangle_{\mathcal{M}}=\int_{0}^{1} \int_{0}^{\infty} h(s) w_{x}(x, s) z_{x}(x, s) d s d x .
$$

As well, we defined the space

$$
\mathcal{D}(\mathcal{M})=\left\{\eta, \eta_{s} \in \mathcal{M}, \eta(x, 0)=0\right\} .
$$

We define the inner product

$$
\begin{aligned}
& \langle(u, \varphi, v, \psi, \theta, \eta),(\widehat{u}, \widehat{\varphi}, \widehat{v}, \widehat{\psi}, \widehat{\theta}, \widehat{\eta})\rangle_{\mathcal{H}} \\
= & \rho_{1} \int_{0}^{1} \varphi \widehat{\varphi} d x+\rho_{2} \int_{0}^{1} \psi \widehat{\psi} d x+\rho_{3} \int_{0}^{1} \theta \widehat{\theta} d x+b_{0} \int_{0}^{1} v_{x} \widehat{v}_{x} d x \\
& +k \int_{0}^{1}\left(u_{x}+v\right)\left(\widehat{u}_{x}+\widehat{v}\right) d x+\int_{0}^{1} \int_{0}^{+\infty} h(s) \eta_{x}(x, s) \widehat{\eta}_{x}(x, s) d s d x,
\end{aligned}
$$


which together with $\mathcal{H}$ form a Hilbert space. Moreover, the domain of the linear operator $A$ is defined by

$$
\mathcal{D}(A):=\left\{\begin{aligned}
U \in \mathcal{H} \mid \bar{u} & \in H^{2}(0,1), \varphi \in H_{*}^{1}(0,1), v \in H^{2}(0,1), \psi \in H_{0}^{1}(0,1), \\
\theta & \in H_{0}^{1}(0,1), \eta \in \mathcal{D}(\mathcal{M}), \eta_{x} \in H^{1}(0,1)
\end{aligned}\right\} .
$$

\section{Wellposedness}

The theorem below is the wellposedness result for problem $1.2-(1.3)$.

Theorem 3.1. Assume (A1) and (A2) hold and $f \in L^{2}(0,1)$. If $U_{0} \in \mathcal{H}$, then the Cauchy problem 2.10 has a unique weak solution

$$
U \in C([0,+\infty) ; \mathcal{H})
$$

Furthermore, if $U_{0} \in \mathcal{D}(A)$, then

$$
U \in C([0,+\infty) ; \mathcal{D}(A)) \cap C^{1}((0,+\infty) ; \mathcal{H}) .
$$

Proof. In other to prove Theorem 3.1, we will show that the linear operator $A$ is monotone and maximal, then further show that the function $F$ is globally Lipschitz. For the maximality and monotonicity of $A$, see [9]. We turn our attention to show that $F$ is Lipschitz. Let $R>0$ and consider

$$
B^{R}=\left\{W=\left(w_{1}, w_{2}, w_{3}, w_{4}, w_{5}, w_{6}\right) \in \mathcal{D}(A):\|W\|_{\mathcal{H}} \leq R\right\} .
$$

Let $W, Z \in B^{R}$, using the embedding of $H_{*}^{1}(0,1)$ in $L^{\infty}(0,1)$ and the fact that $g \in C^{1}(\mathbb{R})$, we have

$$
\begin{aligned}
\|F(W)-F(Z)\|_{\mathcal{H}}^{2} & =\frac{1}{\rho_{2}} \int_{0}^{1}\left|g\left(w_{3}\right)-g\left(z_{3}\right)\right|^{2} d x \\
& \leq \frac{1}{\rho_{2}}\left\|g^{\prime}(\omega)\right\|_{L^{\infty}(0,1)}\left\|w_{3}-z_{3}\right\|_{L^{2}(0,1)}^{2} \leq C(R)\|W-Z\|_{\mathcal{H}}^{2},
\end{aligned}
$$

where $\omega=a w_{3}+(1-a) z_{3}, a \in(0,1)$. Hence $F$ is locally Lipschitz. It follows from Hille-Yosida Theorem, the existence of a local unique weak solution, i.e.,

$$
U \in C\left(\left[0, T_{m}\right) ; \mathcal{H}\right), \quad T_{m}>0 .
$$

We now prove that the solution is indeed global, to do this, we show that $\|U(t)\|_{\mathcal{H}}$ is uniformly bounded independent of time.

Now, we multiply $(2.7)_{1}$ by $\bar{u}_{t}$, then integrate by parts over $(0,1)$ while taking into account the boundary conditions, we obtain

$$
\frac{1}{2} \frac{d}{d t}\left(\rho_{1}\left\|\bar{u}_{t}\right\|^{2}+k\left\|u_{x}+v\right\|^{2}\right)=k\left(u_{x}+v, v_{t}\right)-\left(\theta_{x}, \bar{u}_{t}\right) .
$$


Next, multiply 2.7$)_{2}$ by $v_{t}$, integrate by parts over $(0,1)$, using the boundary conditions and $(2.7)_{4}$, we arrive at

$$
\begin{aligned}
& \frac{1}{2} \frac{d}{d t}\left[\rho_{2}\left\|v_{t}\right\|^{2}+b_{0}\left\|v_{x}\right\|^{2}+2(G(v), 1)+\left\|\eta_{x}\right\|_{\mathcal{M}}^{2}-2(v, f)\right] \\
= & -k\left(u_{x}+v, v_{t}\right)+\gamma\left(\theta, v_{t}\right)+\frac{1}{2} \int_{0}^{1} \int_{0}^{\infty} h^{\prime}(s)\left|\eta_{x}(x, s)\right|^{2} d s d x .
\end{aligned}
$$

Finally, we multiply 2.7$]_{3}$ by $\theta$, integrate by parts over $(0,1)$, and taking into account the boundary conditions, we obtain

$$
\frac{1}{2} \frac{d}{d t}\left(\rho_{3}\|\theta\|^{2}\right)=-\beta\left\|\theta_{x}\right\|^{2}+\gamma\left(\theta_{x}, \bar{u}_{t}\right)-\gamma\left(\theta, v_{t}\right) .
$$

Addition of (3.2)-(3.4) gives

$$
\frac{d}{d t} E(t)=\frac{1}{2} \int_{0}^{1} \int_{0}^{\infty} h^{\prime}(s)\left|\eta_{x}(x, s)\right|^{2} d s d x-\beta\left\|\theta_{x}\right\|^{2} \leq 0, \quad \forall t \geq 0,
$$

where

$$
\begin{aligned}
E(t)= & \frac{1}{2}\left[\rho_{1}\left\|\bar{u}_{t}\right\|^{2}+\rho_{2}\left\|v_{t}\right\|^{2}+\rho_{3}\|\theta\|^{2}+b_{0}\left\|v_{x}\right\|^{2}+\left\|\eta_{x}\right\|_{\mathcal{M}}^{2}+k\left\|u_{x}+v\right\|^{2}\right] \\
& +\frac{1}{2}[2(G(v), 1)-(2 v, f)] .
\end{aligned}
$$

We integrate $(3.5)$ over $(0, t)$ and obtain

$$
E(t)+\beta \int_{0}^{t}\left\|\theta_{x}(s)\right\|^{2} d s \leq E(0), \quad \forall t \geq 0 .
$$

From (2.3), Hölder, Poincaré and Young inequalities, we get

$$
\begin{aligned}
E(t) \geq & \frac{1}{2}\left[\rho_{1}\left\|\bar{u}_{t}\right\|^{2}+\rho_{2}\left\|v_{t}\right\|^{2}+\rho_{3}\|\theta\|^{2}+\left\|\eta_{x}\right\|_{\mathcal{M}}^{2}+k\left\|u_{x}+v\right\|^{2}\right] \\
& +\left(\frac{b_{0}}{2}-\delta-2 \rho \lambda_{1}\right)\left\|v_{x}\right\|^{2}-2 C_{\rho}-c\|f\|^{2} .
\end{aligned}
$$

We choose $\delta$ and $\rho$ small enough such that $b_{0} / 2-\delta-2 \rho \lambda_{1}>0$, hence

$$
E(t) \geq C_{0}\left\|\left(\bar{u}, \bar{u}_{t}, v, v_{t}, \theta, \eta\right)\right\|_{\mathcal{H}}^{2}-C .
$$

Using Hölder, Poincaré, Young inequalities, embedding of $H^{1}(0,1)$ in $L^{\infty}(0,1)$, and (2.4) we have that

$$
\begin{aligned}
-2(v, f) & \leq \delta \lambda_{1}\left\|v_{x}\right\|^{2}+c\|f\|^{2} \\
2(G(v), 1) & \leq c \int_{0}^{1} v^{2}\left|g^{\prime}(\alpha v)\right| d x+c \int_{0}^{1}|v \| g(0)| d x+c \int_{0}^{1} v^{2} d x+C_{\rho}^{\prime \prime} \\
& \leq c \lambda_{1}\left(\left\|g^{\prime}(\alpha v)\right\|_{L^{\infty}(0,1)}+1\right)\left\|v_{x}\right\|^{2}+c,
\end{aligned}
$$


where $\alpha \in(0,1)$. It follows that

$$
\begin{aligned}
E(t) \leq & \frac{1}{2}\left[\rho_{1}\left\|\bar{u}_{t}\right\|^{2}+\rho_{2}\left\|v_{t}\right\|^{2}+\rho_{3}\|\theta\|^{2}+\left\|\eta_{x}\right\|_{\mathcal{M}}^{2}+k\left\|u_{x}+v\right\|^{2}\right] \\
& +\left[\frac{b_{0}}{2}+c \lambda_{1}\left(\delta+\left\|g^{\prime}(\alpha v)\right\|_{L^{\infty}(0,1)}+1\right)\right]\left\|v_{x}\right\|^{2}+c\|f\|^{2}+c .
\end{aligned}
$$

Thus, we have

$$
E(t) \leq c_{0}\left\|\left(\bar{u}, \bar{u}_{t}, v, v_{t}, \theta, \eta\right)\right\|_{\mathcal{H}}^{2}+c,
$$

where $c_{0}=c_{0}\left(\left\|g^{\prime}(\alpha v)\right\|_{L^{\infty}(0,1)}\right)$. Combining 3.6), 3.7) and 3.8), we arrive at

$$
\|U(t)\|_{\mathcal{H}}^{2}+C \int_{0}^{t}\left\|\theta_{x}(s)\right\|^{2} d s \leq c\left(\left\|U_{0}\right\|_{\mathcal{H}}^{2}+1\right) \leq \bar{C}, \quad \forall t \geq 0 .
$$

Therefore, $\|U(t)\|_{\mathcal{H}}^{2}$ is bounded uniformly independent of time. The above computations were obtained for regular solutions. However, by density argument the result remains true for weak solutions. This completes the proof.

\section{Global attractor}

In this section, we turn our attention to proving the existence of the global attractor for the system (2.7). Keeping in mind Theorem 3.1, there exists a solution semi-group

$$
S(t): \mathcal{H} \rightarrow \mathcal{H}
$$

defined by

$$
S(t) U_{0}=U(t), \quad \forall t \geq 0,
$$

where $U$ is the unique solution to system $2.7-2.9$.

Lemma 4.1. The semigroup $S(t)$ is strongly continuous in $\mathcal{H}$.

Proof. Let $U^{j}=\left(\bar{u}^{j}, \bar{u}_{t}^{j}, v^{j}, v_{t}^{j}, \theta^{j}, \eta^{j}\right)^{T}, j=1,2$ be two solutions of system (2.7)-2.9. Then $U=U^{1}-U^{2}$ satisfies

$$
\begin{cases}\rho_{1} u_{t t}-k\left(u_{x}+v\right)_{x}+\gamma \theta_{x}=0, & x \in(0,1), t>0, \\ \rho_{2} v_{t t}-b_{0} v_{x x}+k\left(u_{x}+v\right)-\gamma \theta & \\ \quad-\int_{0}^{+\infty} h(s) \eta_{x x}(x, s) d s+g\left(v^{1}\right)-g\left(v^{2}\right)=0, & x \in(0,1), t>0, \\ \rho_{3} \theta_{t}-\beta \theta_{x x}+\gamma\left(u_{x}+v\right)_{t}=0, & x \in(0,1), t>0, \\ \eta_{t}+\eta_{s}-v_{t}=0, & x \in(0,1), t, s>0\end{cases}
$$

with initial data $U_{0}=U_{0}^{1}-U_{0}^{2}$. 
We multiply 4.1$)_{1}$ by $\left.\bar{u}_{t}, 4.1\right)_{2}$ by $v_{t}$, and 4.1$)_{3}$ by $\theta$, making use of $(4.1)_{4}$, then perform integration by parts over $(0,1)$. Addition of the results from these leads to

$$
\begin{aligned}
& \frac{1}{2} \frac{d}{d t}\left[\rho_{1}\left\|\bar{u}_{t}\right\|^{2}+\rho_{2}\left\|v_{t}\right\|^{2}+b_{0}\left\|v_{x}\right\|^{2}+\rho_{3}\|\theta\|^{2}+\left\|\eta_{x}\right\|_{\mathcal{M}}^{2}+k\left\|u_{x}+v\right\|^{2}\right] \\
& +\left(g\left(v^{1}\right)-g\left(v^{2}\right), v_{t}\right) \\
= & -\beta\left\|\theta_{x}\right\|^{2}+\frac{1}{2} \int_{0}^{1} \int_{0}^{\infty} h^{\prime}(s)\left|\xi_{x}(x, s)\right|^{2} d s d x .
\end{aligned}
$$

Therefore

$$
\begin{aligned}
& \frac{1}{2} \frac{d}{d t}\left[\rho_{1}\left\|\bar{u}_{t}\right\|^{2}+\rho_{2}\left\|v_{t}\right\|^{2}+b_{0}\left\|v_{x}\right\|^{2}+\rho_{3}\|\theta\|^{2}+\left\|\eta_{x}\right\|_{\mathcal{M}}^{2}+k\left\|u_{x}+v\right\|^{2}\right] \\
\leq & -\left(g\left(v^{1}\right)-g\left(v^{2}\right), v_{t}\right) .
\end{aligned}
$$

Performing same computations as in (3.1), we deduce that

$$
\begin{aligned}
& \frac{1}{2} \frac{d}{d t}\left[\rho_{1}\left\|\bar{u}_{t}\right\|^{2}+\rho_{2}\left\|v_{t}\right\|^{2}+b_{0}\left\|v_{x}\right\|^{2}+\rho_{3}\|\theta\|^{2}+\left\|\eta_{x}\right\|_{\mathcal{M}}^{2}+k\left\|u_{x}+v\right\|^{2}\right] \\
\leq & c\left[\rho_{1}\left\|\bar{u}_{t}\right\|^{2}+\rho_{2}\left\|v_{t}\right\|^{2}+\rho_{3}\|\theta\|^{2}+\left\|\eta_{x}\right\|_{\mathcal{M}}^{2}+k\left\|u_{x}+v\right\|^{2}\right],
\end{aligned}
$$

where $c$ is positive constant depending on $U_{0}^{1}$ and $U_{0}^{2}$. Applying Gronwall's lemma to (4.2), we obtain

$$
\|U(t)\|_{\mathcal{H}}^{2} \leq e^{c t}\left(\left\|U_{0}\right\|_{\mathcal{H}}^{2}\right), \quad \forall t \geq 0 .
$$

This completes the proof.

At this point, we recall some standard definitions and theorems that will be used to prove the existence of the global attractor.

Definition 4.2. Let $X$ be a Banach space. A set $\mathscr{B} \subset X$ is an absorbing set for the semigroup $S(t): X \rightarrow X$ if for any bounded set $B \subset X$ there exists a time $t_{0}(B)$ such that $S(t) B \subset \mathscr{B}$ for all $t \geq t_{0}(B)$.

Definition 4.3. A compact subset $\mathcal{A}$ of $H$ is the global attractor for a semigroup $S(t)$ acting on a Hilbert space $H$ if the following conditions are satisfied.

(i) $\mathcal{A}$ is invariant for $S(t)$; i.e.,

$$
S(t) \mathcal{A}=\mathcal{A}, \quad \forall t \geq 0
$$

(ii) $\mathcal{A}$ attracts any bounded subset of $H$; i.e.,

$$
\lim _{t \rightarrow \infty} d_{H}(S(t) B, \mathcal{A})=0, \quad \forall B \subset H
$$

where $d_{H}$ is the Hausdorff semi-distance defined by

$$
d_{H}(A, B)=\sup _{a \in A} \inf _{b \in B}\|a-b\|_{H} .
$$

Theorem 4.4. 5 Let $S(t)$ be a dissipative semigroup on a metric space $H$. Then, $S(t)$ has a compact global attractor in $H$ if and only if it is asymptotically smooth in $H$. 


\subsection{Absorbing set}

We will now show that the semigroup generated by the system (2.7)-2.9 possesses an absorbing set. We prove some technical lemmas as follows.

Lemma 4.5. We define the functional $I_{1}(t)$ by

$$
I_{1}(t)=-\rho_{3} \int_{0}^{1} \theta \int_{0}^{x} \bar{u}_{t}(y) d y d x
$$

$I_{1}(t)$ along the solution of system (2.7)-2.9), satisfies the estimate

$$
I_{1}^{\prime}(t) \leq-\frac{\gamma}{2}\left\|\bar{u}_{t}\right\|^{2}+\varepsilon_{1}\left\|u_{x}+v\right\|^{2}+c\left(1+\frac{1}{\varepsilon_{1}}\right)\left\|\theta_{x}\right\|^{2}+c\left\|v_{t}\right\|^{2}
$$

for any $\varepsilon_{1}>0$.

Proof. We differentiate $I_{1}(t)$ with respect to t, then make use of $(2.7)_{1}$ and $(2.7)_{3}$ and recalling that $m(\bar{u})=0$, we obtain

$$
\begin{aligned}
I_{1}^{\prime}(t)= & -\rho_{3} \int_{0}^{1} \theta \int_{0}^{x} u_{t t}(y) d y d x-\rho_{3} \int_{0}^{1} \theta_{t} \int_{0}^{x} \bar{u}_{t}(y) d y d x \\
= & -\frac{k \rho_{3}}{\rho_{1}} \int_{0}^{1} \theta\left(u_{x}+v\right) d x+\frac{\gamma \rho_{3}}{\rho_{1}}\|\theta\|^{2}+\beta \int_{0}^{1} \theta_{x} \bar{u}_{t} d x \\
& -\gamma\left\|\bar{u}_{t}\right\|^{2}+\gamma \int_{0}^{1} v_{t} \int_{0}^{x} \bar{u}_{t}(y) d y d x .
\end{aligned}
$$

Using Poincaré and Young inequalities, we get for any $\varepsilon_{2}$,

$$
\begin{aligned}
I_{1}^{\prime}(t) \leq & \varepsilon_{2}\left\|u_{x}+v\right\|^{2}+\frac{c}{\varepsilon_{2}}\left\|\theta_{x}\right\|^{2}+c\left\|\theta_{x}\right\|^{2}+\frac{\gamma}{4}\left\|\bar{u}_{t}\right\|^{2}-\gamma\left\|\bar{u}_{t}\right\|^{2} \\
& +c \gamma\left\|v_{t}\right\|^{2}+\frac{\gamma}{4} \int_{0}^{1}\left(\int_{0}^{x} \bar{u}_{t}(y) d y\right)^{2} d x
\end{aligned}
$$

Using Cauchy-Schwarz inequality, the last term in 4.4 is estimated as

$$
\begin{aligned}
\frac{\gamma}{4} \int_{0}^{1}\left(\int_{0}^{x} \bar{u}_{t}(y) d y\right)^{2} d x & \leq \frac{\gamma}{4} \int_{0}^{1}\left(\int_{0}^{x} 1^{2} d y\right)\left(\int_{0}^{x} \bar{u}_{t}^{2}(y) d y\right) d x \\
& \leq \frac{\gamma}{4} \int_{0}^{1}\left(\int_{0}^{1} d x\right)\left(\int_{0}^{1} \bar{u}_{t}^{2}(x) d x\right) d x=\frac{\gamma}{4} \int_{0}^{1} \bar{u}_{t}^{2}(x) d x .
\end{aligned}
$$

Hence (4.4) gives

$$
I_{1}^{\prime}(t) \leq-\frac{\gamma}{2}\left\|\bar{u}_{t}\right\|^{2}+\varepsilon_{2}\left\|u_{x}+v\right\|^{2}+c\left(1+\frac{1}{\varepsilon_{2}}\right)\left\|\theta_{x}\right\|^{2}+c\left\|v_{t}\right\|^{2} .
$$


Lemma 4.6. We define the functional $I_{2}(t)$ by

$$
I_{2}(t)=-\rho_{1} \int_{0}^{1}\left(u_{x}+v\right) \int_{0}^{x} \bar{u}_{t}(y) d y d x
$$

$I_{2}(t)$ along the solution of system $2.7-2.9$, satisfies the estimate

$$
I_{2}^{\prime}(t) \leq-\frac{k}{2}\left\|u_{x}+v\right\|^{2}+c\left\|\theta_{x}\right\|^{2}+c\left\|\bar{u}_{t}\right\|^{2}+c\left\|v_{t}\right\|^{2} .
$$

Proof. We differentiate $I_{2}(t)$ with respect to $t$, then making use of $(2.7)_{1}$ and noting that $m(\bar{u})=0$, we obtain

$$
\begin{aligned}
I_{2}^{\prime}(t) & =-\rho_{1} \int_{0}^{1}\left(u_{x}+v\right) \int_{0}^{x} u_{t t}(y) d y d x-\rho_{1} \int_{0}^{1}\left(u_{x}+v\right)_{t} \int_{0}^{x} \bar{u}_{t}(y) d y d x \\
& =-k\left\|u_{x}+v\right\|^{2}+\gamma \int_{0}^{1}\left(u_{x}+v\right) \theta d x+\rho_{1}\left\|\bar{u}_{t}\right\|^{2}-\rho_{1} \int_{0}^{1} v_{t} \int_{0}^{x} \bar{u}_{t}(y) d y d x .
\end{aligned}
$$

Using Poincaré, Young and Cauchy-Schwarz inequalities, we get

$$
\begin{aligned}
I_{2}^{\prime}(t) & \leq-\frac{k}{2}\left\|u_{x}+v\right\|^{2}+c\left\|\theta_{x}\right\|^{2}+\rho_{1}\left\|\bar{u}_{t}\right\|^{2}+c\left\|v_{t}\right\|^{2}+c \int_{0}^{1}\left(\int_{0}^{x} \bar{u}_{t}(y) d y\right)^{2} d x \\
& \leq-\frac{k}{2}\left\|u_{x}+v\right\|^{2}+c\left\|\theta_{x}\right\|^{2}+c\left\|\bar{u}_{t}\right\|^{2}+c\left\|v_{t}\right\|^{2} .
\end{aligned}
$$

Lemma 4.7. We define the functional $I_{3}(t)$ by

$$
I_{3}(t)=-\rho_{2} \int_{0}^{1} v_{t} \int_{0}^{+\infty} h(s) \eta(x, s) d s d x .
$$

$I_{3}(t)$ along the solution of system (2.7)-2.9), satisfies the estimate

$$
\begin{aligned}
\frac{\partial}{\partial t} I_{3}(t) \leq & -\frac{\rho_{2}\left(b-b_{0}\right)}{2}\left\|v_{t}\right\|^{2}+\varepsilon_{1}\left\|v_{x}\right\|^{2}+\varepsilon_{2}\left\|u_{x}+v\right\|^{2}+\varepsilon_{2}\left\|\theta_{x}\right\|^{2} \\
& +\varepsilon_{2}\|f\|^{2}+\varepsilon_{2}|g(0)|^{2}+c\left[1+\frac{1}{\varepsilon_{2}}+\frac{1}{\varepsilon_{1}}\left(1+\left\|g^{\prime}(\alpha v)\right\|_{L^{\infty}(0,1)}^{2}\right)\right]\left\|\eta_{x}\right\|_{\mathcal{M}}^{2} \\
& -c \int_{0}^{1} \int_{0}^{+\infty} h^{\prime}(s)\left|\eta_{x}(x, s)\right|^{2} d s d x
\end{aligned}
$$

for any $\varepsilon_{1}, \varepsilon_{2}>0$, where $\alpha \in(0,1)$.

Proof. We differentiate $I_{3}(t)$ with respect to $t$, then making use of $(2.7)_{4}$, we obtain

$$
\begin{aligned}
\frac{\partial}{\partial t} I_{3}(t) & =-\rho_{2} \int_{0}^{1} v_{t t} \int_{0}^{+\infty} h(s) \eta(x, s) d s d x-\rho_{2} \int_{0}^{1} v_{t} \int_{0}^{+\infty} h(s) \eta_{t}(x, s) d s d x \\
& =-\rho_{2} \int_{0}^{1} v_{t t} \int_{0}^{+\infty} h(s) \eta(x, s) d s d x-\rho_{2} \int_{0}^{1} v_{t} \int_{0}^{+\infty} h(s)\left(v_{t}(t)-\eta_{s}(x, s)\right) d s d x \\
& =-\rho_{2}\left(b-b_{0}\right)\left\|v_{t}\right\|^{2}-\rho_{2} \int_{0}^{1} v_{t t} \int_{0}^{+\infty} h(s) \eta(x, s) d s d x
\end{aligned}
$$




$$
\begin{aligned}
& +\rho_{2} \int_{0}^{1} v_{t}\left[\left.h(s) \eta(x, s)\right|_{0} ^{+\infty}-\int_{0}^{+\infty} h^{\prime}(s) \eta(x, s) d s\right] d x \\
= & -\rho_{2}\left(b-b_{0}\right)\left\|v_{t}\right\|^{2}-\rho_{2} \int_{0}^{1} v_{t t} \int_{0}^{+\infty} h(s) \eta(x, s) d s d x \\
& -\rho_{2} \int_{0}^{1} v_{t} \int_{0}^{+\infty} h^{\prime}(s) \eta(x, s) d s d x .
\end{aligned}
$$

Now, making use of $(2.7)_{2}$ and integration by parts, we arrive at

$$
\begin{aligned}
\frac{\partial}{\partial t} I_{3}(t)= & -\rho_{2}\left(b-b_{0}\right)\left\|v_{t}\right\|^{2}-\underbrace{\rho_{2} \int_{0}^{1} v_{t} \int_{0}^{+\infty} h^{\prime}(s) \eta(x, s) d s d x}_{J_{1}} \\
& +\underbrace{b_{0} \int_{0}^{1} v_{x} \int_{0}^{+\infty} h(s) \eta_{x}(x, s) d s d x}_{J_{2}}+\underbrace{k \int_{0}^{1}\left(u_{x}+v\right) \int_{0}^{+\infty} h(s) \eta(x, s) d s d x}_{J_{3}} \\
& -\underbrace{\gamma \int_{0}^{1} \theta \int_{0}^{+\infty} h(s) \eta(x, s) d s d x}_{J_{4}}+\underbrace{\int_{0}^{1}\left(\int_{0}^{+\infty} h(s) \eta_{x}(x, s) d s\right)^{2} d x}_{J_{6}} \\
& +\underbrace{\int_{0}^{1} g(v) \int_{0}^{+\infty} h(x, s) d s d x}_{J_{5}}-\underbrace{\int_{0}^{1}}_{J_{7}^{1} f(x) \int_{0}^{+\infty} h(s) \eta(x, s) d s d x} .
\end{aligned}
$$

Using Poincaré, Young and Cauchy-Schwarz inequalities, we estimate $J_{1}, J_{2}, \ldots, J_{7}$ as follows:

$$
\begin{aligned}
J_{1}(t) & \leq \frac{\rho_{2}\left(b-b_{0}\right)}{2}\left\|v_{t}\right\|^{2}-c h(0) \int_{0}^{1} \int_{0}^{+\infty} h^{\prime}(s)\left|\eta_{x}(x, s)\right|^{2} d s d x, \\
J_{2}(t) & \leq \frac{\varepsilon_{1}}{2}\left\|v_{x}\right\|^{2}+\frac{c\left(b-b_{0}\right)}{\varepsilon_{1}} \int_{0}^{1} \int_{0}^{+\infty} h(s)\left|\eta_{x}(x, s)\right|^{2} d s d x, \\
J_{3}(t) & \leq \varepsilon_{1}\left\|u_{x}+v\right\|^{2}+\frac{c\left(b-b_{0}\right) \lambda_{1}}{\varepsilon_{1}} \int_{0}^{1} \int_{0}^{+\infty} h(s)\left|\eta_{x}(x, s)\right|^{2} d s d x, \\
J_{4}(t) & \leq \varepsilon_{2}\left\|\theta_{x}\right\|^{2}+\frac{c\left(b-b_{0}\right) \lambda_{1}}{\varepsilon_{2}} \int_{0}^{1} \int_{0}^{+\infty} h(s)\left|\eta_{x}(x, s)\right|^{2} d s d x, \\
J_{5}(t) & \leq \int_{0}^{1} \int_{0}^{+\infty} \sqrt{h(s)}\left[\sqrt{h(s)} \eta_{x}(x, s)\right] d s d x \\
& \leq \int_{0}^{1}\left(\int_{0}^{+\infty} h(s) d s\right)\left(\int_{0}^{+\infty} h(s) \eta_{x}^{2}(x, s) d s\right) d x \\
& \leq\left(b-b_{0}\right) \int_{0}^{1} \int_{0}^{+\infty} h(s)\left|\eta_{x}(x, s)\right|^{2} d s d x, \\
J_{6}(t) & =\int_{0}^{1}(g(v)-g(0)) \int_{0}^{+\infty} h(s) \eta(x, s) d s d x+\int_{0}^{1}-g(0) \int_{0}^{+\infty} h(s) \eta(x, s) d s d x \\
& \leq \int_{0}^{1} g^{\prime}(\alpha v) v \int_{0}^{+\infty} h(s) \eta(x, s) d s d x+\varepsilon_{2}|g(0)|^{2}
\end{aligned}
$$




$$
\begin{aligned}
& +\frac{c}{\varepsilon_{2}} \int_{0}^{1}\left(\int_{0}^{+\infty} h(s) \eta(x, s) d s\right)^{2} d x \\
\leq & \frac{\varepsilon_{1}}{2}\left\|v_{x}\right\|^{2}+\frac{c\left(b-b_{0}\right) \lambda_{1}\left(1+\left\|g^{\prime}(\alpha v)\right\|_{L^{\infty}(0,1)}^{2}\right)}{\varepsilon_{1}} \int_{0}^{1} \int_{0}^{+\infty} h(s)\left|\eta_{x}(x, s)\right|^{2} d s d x \\
& +\varepsilon_{2}|g(0)|^{2}, \\
J_{7}(t) \leq & \varepsilon_{2}\|f\|^{2}+\frac{c\left(b-b_{0}\right) \lambda_{1}}{\varepsilon_{2}} \int_{0}^{1} \int_{0}^{+\infty} h(s)\left|\eta_{x}(x, s)\right|^{2} d s d x .
\end{aligned}
$$

Substituting $J_{1}, J_{2}, \ldots, J_{7}$ in (4.7), the expected estimate (4.6) follows immediately.

Lemma 4.8. We define the functional $I_{4}(t)$ by

$$
I_{4}(t)=\rho_{2} \int_{0}^{1} v v_{t} d x .
$$

$I_{4}(t)$ along the solution of system (2.7)-2.9), satisfies the estimate

$$
I_{4}^{\prime}(t) \leq-\frac{b_{0}}{4}\left\|v_{x}\right\|^{2}+\rho_{2}\left\|v_{t}\right\|^{2}+c\left\|u_{x}+v\right\|^{2}+c\left\|\theta_{x}\right\|^{2}+c\|f\|^{2}+\left(b-b_{0}\right)\left\|\eta_{x}\right\|_{\mathcal{M}}^{2}+c_{\rho}^{\prime \prime} .
$$

Proof. We differentiate $I_{4}(t)$ with respect to $t$, then make use of $(2.7)_{2}$, we obtain

$$
\begin{aligned}
I_{4}^{\prime}(t)= & \rho_{2} \int_{0}^{1} v_{t}^{2} d x+\rho_{2} \int_{0}^{1} v v_{t t} d x \\
= & \rho_{2}\left\|v_{t}\right\|^{2}-b_{0}\left\|v_{x}\right\|^{2}-k \int_{0}^{1}\left(u_{x}+v\right) v+\gamma \int_{0}^{1} v \theta \\
& -\int_{0}^{1} v_{x} \int_{0}^{+\infty} h(s) \eta_{x}(x, s) d s d x-\int_{0}^{1} v g(v) d x+\int_{0}^{1} v f(x) d x .
\end{aligned}
$$

Next, using Poincaré and Young inequalities, we obtain

$$
\begin{aligned}
I_{4}^{\prime}(t) \leq & -\frac{b_{0}}{2}\left\|v_{x}\right\|^{2}+\rho_{2}\left\|v_{t}\right\|^{2}+c\left\|u_{x}+v\right\|^{2}+c\left\|\theta_{x}\right\|^{2}+c\|f\|^{2} \\
& -\int_{0}^{1} v g(v) d x+c \int_{0}^{1}\left(\int_{0}^{+\infty} h(s) \eta_{x}(x, s) d s\right)^{2} d x .
\end{aligned}
$$

Now, taking into account Cauchy-Schwarz inequality and condition (2.4), we deduce

$$
\begin{gathered}
\int_{0}^{1}\left(\int_{0}^{+\infty} h(s) \eta_{x}(x, s) d s\right)^{2} d x \leq\left(b-b_{0}\right)\left\|\eta_{x}\right\|_{\mathcal{M}}^{2}, \\
-\int_{0}^{1} v g(v) d x \leq\left(C_{1}+1\right) \lambda_{1} \rho\left\|v_{x}\right\|^{2}+C_{\rho}^{\prime}+C_{\rho} \quad \text { for any } \rho>0 .
\end{gathered}
$$

Hence the estimate 4.9 yields

$$
\begin{aligned}
I_{4}^{\prime}(t) \leq & -\left(\frac{b_{0}}{2}-c \rho\right)\left\|v_{x}\right\|^{2}+\rho_{2}\left\|v_{t}\right\|^{2}+c\left\|u_{x}+v\right\|^{2}+c\left\|\theta_{x}\right\|^{2}+c\|f\|^{2} \\
& +\left(b-b_{0}\right)\left\|\eta_{x}\right\|_{\mathcal{M}}^{2}+C_{\rho}^{\prime}+C_{\rho} .
\end{aligned}
$$

We choose $\rho$ small enough such that $b_{0} / 2-c \rho \geq b_{0} / 4$ and 4.8 follows immediately. 
Let the functional $\mathscr{L}$ be defined as

$$
\mathscr{L}(t)=N E(t)+N_{1} I_{1}(t)+N_{2} I_{2}(t)+N_{3} I_{3}(t)+I_{4}(t),
$$

where $N, N_{1}, N_{2}$ and $N_{3}$ are suitable positive constants to be determined later.

Lemma 4.9. There exist positive constants $\varrho_{1}, \varrho_{2}, \varrho_{3}$ and $\varrho_{4}$, such that the functional $\mathscr{L}(t)$ along the solution of system (2.7)-2.9), satisfies

$$
\varrho_{1}\left\|\left(\bar{u}, \bar{u}_{t}, v, v_{t}, \theta, \eta\right)(t)\right\|_{\mathcal{H}}^{2}-\varrho_{2} \leq \mathscr{L}(t) \leq \varrho_{3}\left\|\left(\bar{u}, \bar{u}_{t}, v, v_{t}, \theta, \eta\right)(t)\right\|_{\mathcal{H}}^{2}+\varrho_{4}, \quad \forall t \geq 0
$$

where $\varrho_{3}=\varrho_{3}\left(\left\|g^{\prime}(\alpha v)\right\|_{L^{\infty}(0,1)}\right)$.

Proof. On the one hand, using Hölder, Poincaré, Young and Cauchy-Schwarz inequalities, we have

$$
\begin{aligned}
\mathscr{L}(t) \leq & N E(t)+N_{1}\left|I_{1}(t)\right|+N_{2}\left|I_{2}(t)\right|+N_{3}\left|I_{3}(t)\right|+\left|I_{4}(t)\right| \\
\leq & \frac{N \rho_{1}}{2}\left\|\bar{u}_{t}\right\|^{2}+\left(N_{1} \rho_{3}^{2}+\frac{N \rho_{3}}{2}\right)\|\theta\|^{2}+c\left(N_{1}+N_{2}\right) \int_{0}^{1}\left(\int_{0}^{x} \bar{u}_{t}(y) d y\right)^{2} d x \\
& +\left(N_{2} \rho_{1}^{2}+\frac{N k}{2}\right)\left\|u_{x}+v\right\|^{2}+\left(c+N_{3} \rho_{2}^{2}+\frac{N \rho_{2}}{2}\right)\left\|v_{t}\right\|^{2} \\
& +N \int_{0}^{1} G(v) d x+\left(\rho_{2}^{2} \lambda_{1}+\frac{N b_{0}}{2}\right)\left\|v_{x}\right\|^{2}+\frac{N}{2}\left\|\eta_{x}\right\|_{\mathcal{M}}^{2} \\
& +c N_{3} \int_{0}^{1}\left(\int_{0}^{+\infty} h(s) \eta(x, s) d s\right)^{2} d x+\int_{0}^{1}|v f| d x .
\end{aligned}
$$

Using assumption (2.4), we have

$$
\begin{aligned}
\int_{0}^{1} G(v) d x & \leq \frac{1}{C_{1}} \int_{0}^{1}\left(v g(v)+\rho v^{2}\right) d x+\frac{C_{\rho}^{\prime}}{C_{1}} \\
& \leq c \int_{0}^{1}|v|^{2} d x+\int_{0}^{1}|v|(|g(v)-g(0)|) d x+\int_{0}^{1}|v \| g(0)| d x \\
& \leq c \lambda_{1}\left(\left\|g^{\prime}(\alpha v)\right\|_{L^{\infty}(0,1)}^{2}+1\right)\left\|v_{x}\right\|^{2}+C, \quad \alpha \in(0,1) .
\end{aligned}
$$

Also,

$$
\int_{0}^{1}|v f| d x \leq \lambda_{1}\left\|v_{x}\right\|^{2}+c\|f\|^{2} .
$$

Hence, from 4.12 and 4.13, the estimate 4.11 yields

$$
\mathscr{L}(t) \leq c\left(\rho_{1}\left\|\bar{u}_{t}\right\|^{2}+\rho_{2}\left\|v_{t}\right\|^{2}+b_{0}\left\|v_{x}\right\|^{2}+\rho_{3}\|\theta\|^{2}+\left\|\eta_{x}\right\|_{\mathcal{M}}^{2}+k\left\|u_{x}+v\right\|^{2}\right)+C,
$$


where $c=c\left(\left\|g^{\prime}(\alpha v)\right\|_{L^{\infty}(0,1)}\right)$. On the other hand, we have

$$
\begin{aligned}
\mathscr{L}(t) \geq & \frac{N}{2}\left(\rho_{1}\left\|\bar{u}_{t}\right\|^{2}+\rho_{2}\left\|v_{t}\right\|^{2}+b_{0}\left\|v_{x}\right\|^{2}+\rho_{3}\|\theta\|^{2}+\left\|\eta_{x}\right\|_{\mathcal{M}}^{2}+k\left\|u_{x}+v\right\|^{2}\right) \\
& +\frac{N}{2}\left(-2 \rho \lambda_{1}\left\|v_{x}\right\|^{2}-2 C_{\rho}-\delta_{1} \lambda_{1}-C_{\delta_{1}}\|f\|^{2}\right)-N_{1} \rho_{3}\left(\frac{1}{2}\|\theta\|^{2}+\frac{1}{2}\left\|\bar{u}_{t}\right\|^{2}\right) \\
& -N_{2} \rho_{1}\left(\frac{1}{2}\left\|u_{x}+v\right\|^{2}+\frac{1}{2}\left\|\bar{u}_{t}\right\|^{2}\right)-N_{3} \rho_{2}\left(\frac{1}{2}\left\|v_{t}\right\|^{2}+\frac{\left(b-b_{0}\right) \lambda_{1}}{2}\left\|\eta_{x}\right\|_{\mathcal{M}}^{2}\right) \\
& -\rho_{2}\left(\frac{1}{2}\left\|v_{t}\right\|^{2}+\frac{\lambda_{1}}{2}\left\|v_{x}\right\|^{2}\right) .
\end{aligned}
$$

Hence, we deduce

$$
\begin{aligned}
\mathscr{L}(t) \geq & {\left[\frac{N}{2}-\frac{N_{1} \rho_{3}}{2 \rho_{1}}-\frac{N_{2}}{2}\right] \rho_{1}\left\|\bar{u}_{t}\right\|^{2}+\left[\frac{N}{2}-\frac{N_{3}}{2}-\frac{1}{2}\right] \rho_{2}\left\|v_{t}\right\|^{2}+\left[\frac{N}{2}-\frac{N_{1}}{2}\right] \rho_{3}\|\theta\|^{2} } \\
& +\left[N\left(\frac{1}{2}-\frac{2 \rho \lambda_{1}}{b_{0}}-\frac{\delta_{1} \lambda_{1}}{b_{0}}\right)-\frac{\rho_{1} \lambda_{1}}{2 b_{0}}\right] b_{0}\left\|v_{x}\right\|^{2}+\left[\frac{N}{2}-N_{3} \frac{\rho_{2}\left(b-b_{0}\right) \lambda_{1}}{2}\right]\left\|\eta_{x}\right\|_{\mathcal{M}}^{2} \\
& +\left[\frac{N}{2}-\frac{N_{1} \rho_{1}}{2 k}\right] k\left\|u_{x}+v\right\|^{2}-\left(\frac{N}{2} C_{\delta_{1}}\|f\|^{2}+N C_{\rho}\right) .
\end{aligned}
$$

Now, we choose $\rho$ and $\delta_{1}$ small such that

$$
\frac{1}{2}-\frac{2 \rho \lambda_{1}}{b_{0}}-\frac{\delta_{1} \lambda_{1}}{b_{0}}>0
$$

then we choose $N$ large enough so that

$$
\begin{gathered}
\frac{N}{2}-\frac{N_{1} \rho_{3}}{2 \rho_{1}}-\frac{N_{2}}{2}>0, \frac{N}{2}-\frac{N_{3}}{2}-\frac{1}{2}>0, \frac{N}{2}-\frac{N_{1}}{2}>0, \\
N\left(\frac{1}{2}-\frac{2 \rho \lambda_{1}}{b_{0}}-\frac{\delta_{1} \lambda_{1}}{b_{0}}\right)-\frac{\rho_{1} \lambda_{1}}{2 b_{0}}>0, \frac{N}{2}-N_{3} \frac{\rho_{2}\left(b-b_{0}\right) \lambda_{1}}{2}>0 \text { and } \frac{N}{2}-\frac{N_{1} \rho_{1}}{2 k}>0 .
\end{gathered}
$$

It follows that

$$
\mathscr{L}(t) \geq \widetilde{c}\left(\rho_{1}\left\|\bar{u}_{t}\right\|^{2}+\rho_{2}\left\|v_{t}\right\|^{2}+b_{0}\left\|v_{x}\right\|^{2}+\rho_{3}\|\theta\|^{2}+\left\|\eta_{x}\right\|_{\mathcal{M}}^{2}+k\left\|u_{x}+v\right\|^{2}\right)-\widetilde{C} .
$$

Therefore, the inequality 4.10 immediately follows from 4.14 and 4.15.

Lemma 4.10. There exist two positive constants $\alpha_{1}$ and $\alpha_{2}$, such that the functional $\mathscr{L}(t)$ along the solution of system (2.7)-2.9), satisfies

$$
\frac{d}{d t} \mathscr{L}(t)+\alpha_{1} \mathscr{L}(t) \leq \alpha_{2}, \quad \forall t \geq 0
$$

where $\alpha_{1}=\alpha_{1}\left(\left\|g^{\prime}(\alpha v)\right\|_{L^{\infty}(0,1)}\right)$. 
Proof. Keeping in mind (3.5), 4.3), 4.5), 4.6) and (4.8), we have

$$
\begin{aligned}
\frac{d}{d t} \mathscr{L}(t) \leq & -\left[\frac{N_{1} \gamma}{2}-N_{2} c\right]\left\|\bar{u}_{t}\right\|^{2}-\left[\frac{\rho_{2}\left(b-b_{0}\right)}{2} N_{3}-c N_{1}-c N_{2}-\rho_{2}\right]\left\|v_{t}\right\|^{2} \\
& -\left[N \beta-c\left(1+\frac{1}{\varepsilon_{2}}\right) N_{1}-c N_{2}-\varepsilon_{2} N_{3}+c\right]\left\|\theta_{x}\right\|^{2}-\left[\frac{b_{0}}{4}-\varepsilon_{1} N_{3}\right]\left\|v_{x}\right\|^{2} \\
& -\left[N_{2} \frac{k}{2}-\varepsilon_{2} N_{1}-\varepsilon_{1} N_{3}-c\right]\left\|u_{x}+v\right\|^{2} \\
& +\left[c N_{3}\left(1+\frac{1}{\varepsilon_{2}}+\frac{1}{\varepsilon_{1}}\left(1+\left\|g^{\prime}(\alpha v)\right\|_{L^{\infty}(0,1)}^{2}\right)\right)+\left(b-b_{0}\right)\right]\left\|\eta_{x}\right\|_{\mathcal{M}}^{2} \\
& +\left[\frac{N}{2}-c N_{3}\right] \int_{0}^{1} \int_{0}^{+\infty} h^{\prime}(s)\left|\eta_{x}(x, s)\right|^{2} d s d x+C .
\end{aligned}
$$

We take $\varepsilon_{1}=b_{0} /\left(8 N_{3}\right)$ and $\varepsilon_{2}=k N_{2} /\left(4 N_{1}\right)$, then using 2.1), we obtain

$$
\begin{aligned}
\frac{d}{d t} \mathscr{L}(t) \leq & -\left[\frac{N_{1} \gamma}{2}-N_{2} c\right]\left\|\bar{u}_{t}\right\|^{2}-\left[\frac{\rho_{2}\left(b-b_{0}\right)}{2} N_{3}-c N_{1}-c N_{2}-\rho_{2}\right]\left\|v_{t}\right\|^{2} \\
& -\left[N \beta-c\left(1+\frac{4 N_{1}}{k N_{2}}\right) N_{1}-\left(c+\frac{k N_{3}}{4 N_{1}}\right) N_{2}-c\right]\left\|\theta_{x}\right\|^{2} \\
& -\frac{b_{0}}{8}\left\|v_{x}\right\|^{2}-\left[N_{2} \frac{k}{4}-\frac{b_{0}}{8}-c\right]\left\|u_{x}+v\right\|^{2} \\
& -\left[N \frac{\xi}{2}-c N_{3}\left(1+\xi+\frac{4 N_{1}}{k N_{2}}+\frac{8 N_{3}}{b_{0}}\left(1+\left\|g^{\prime}(\alpha v)\right\|_{L^{\infty}(0,1)}^{2}\right)\right)-\left(b-b_{0}\right)\right]\left\|\eta_{x}\right\|_{\mathcal{M}}^{2} \\
& +C .
\end{aligned}
$$

Now, we choose $N_{2}$ large enough such that

$$
N_{2} \frac{k}{4}-\frac{b_{0}}{8}-c>0
$$

then choose $N_{1}$ large enough such that

$$
\frac{N_{1} \gamma}{2}-N_{2} c>0
$$

We can then choose $N_{3}$ large enough such that

$$
\frac{\rho_{2}\left(b-b_{0}\right)}{2} N_{3}-c N_{1}-c N_{2}-\rho_{2}>0,
$$

finally we choose $N$ large enough such that

$$
N \beta-c\left(1+\frac{4 N_{1}}{k N_{2}}\right) N_{1}-\left(c+\frac{k N_{3}}{4 N_{1}}\right) N_{2}-c>0
$$

and

$$
N \frac{\xi}{2}-c N_{3}\left(1+\xi+\frac{4 N_{1}}{k N_{2}}+\frac{8 N_{3}}{b_{0}}\left(1+\left\|g^{\prime}(\alpha v)\right\|_{L^{\infty}(0,1)}^{2}\right)\right)-\left(b-b_{0}\right)>0
$$


We deduce that

$$
\frac{d}{d t} \mathscr{L}(t) \leq-\beta_{1}\left\|\left(\bar{u}, \bar{u}_{t}, v, v_{t}, \theta, \eta\right)\right\|_{\mathcal{H}}^{2}+\beta_{2}
$$

for some positive constants $\beta_{1}$ and $\beta_{2}$, where $\beta_{1}=\beta_{1}\left(\left\|g^{\prime}(\alpha v)\right\|_{L^{\infty}(0,1)}\right)$. Due to (4.10) and 4.17, we obtain 4.16.

Theorem 4.11. Let the assumptions of Theorem 3.1 hold, then the semigroup $S(t)$ of system 2.7)-2.9), possesses a bounded absorbing set $\mathscr{B}$ in $(\mathcal{H}, S(t))$.

Proof. We integrate 4.16) over $(0, t)$ and we obtain

$$
\mathscr{L}(t) \leq \mathscr{L}(0) e^{-\alpha_{1} t}+\alpha_{2}\left(1-e^{-\alpha_{1} t}\right) \leq \mathscr{L}(0) e^{-\alpha_{1} t}+\alpha_{2}
$$

Using (4.10, we get

$$
\begin{aligned}
\left\|\left(\bar{u}, \bar{u}_{t}, v, v_{t}, \theta, \xi\right)\right\|_{\mathcal{H}}^{2} & \leq \frac{1}{\varrho_{1}} \mathscr{L}(0) e^{-\alpha_{1} t}+\frac{1}{\varrho_{1}}\left(\alpha_{2}+\varrho_{2}\right) \\
& \leq C(R)\left\|\left(\bar{u}_{0}, \bar{u}_{1}, v_{0}, v_{1}, \theta_{0}, \xi^{0}\right)\right\|_{\mathcal{H}}^{2}+\frac{1}{\varrho_{1}}\left(\alpha_{2}+\varrho_{2}+\varrho_{4}\right) .
\end{aligned}
$$

Therefore, for $R>\sqrt{\frac{1}{\mu_{1}}\left(\alpha_{2}+\varrho_{2}+\varrho_{4}\right)}$, the ball $\mathscr{B}(0, R)$ is a bounded absorbing set in $(\mathcal{H}, S(t))$. This completes the proof.

\subsection{Asymptotic smoothness of the semigroup $S(t)$}

We will now establish the asymptotic smoothness of the semigroup $S(t)$ generated by system 2.7$)-(2.9)$ in $\mathcal{H}$. The following lemma will be used in the sequel.

Lemma 4.12. 5 Let $H$ be a Banach space. Assume that for any $\mathcal{B} \subset H$ bounded and positively invariant and for any $\epsilon>0$, there exists $T=T(\epsilon, \mathcal{B})$ such that

$$
\left\|S(T) y_{1}-S(T) y_{2}\right\|_{H} \leq \epsilon+\Psi_{T}\left(y_{1}, y_{2}\right), \quad \forall y_{1}, y_{2} \in \mathcal{B}
$$

where $\Psi_{T}: H \times H \rightarrow \mathbb{R}$ satisfies, for any sequence $\left\{y_{n}\right\} \subset \mathcal{B}$,

$$
\lim _{j \rightarrow \infty} \lim _{k \rightarrow \infty} \Psi_{T}\left(y_{n_{j}}, y_{n_{k}}\right)=0
$$

Then $S(t)$ is asymptotically smooth.

Let $U^{j}=\left(\bar{u}^{j}, \bar{u}_{t}^{j}, v^{j}, v_{t}^{j}, \theta^{j}, \eta^{j}\right)^{T}, j=1,2$ be two solutions of system 2.7)-(2.9), with corresponding initial data $U_{0}^{j}=\left(\bar{u}_{0}^{j}, \bar{u}_{1}^{j}, v_{0}^{j}, v_{1}^{j}, \theta_{0}^{j}, \eta^{0 j}\right)^{T} \in \mathcal{B}, j=1,2$, where $\mathcal{B} \subset \mathcal{H}$ is 
a bounded and positive invariant set for the semigroup $S(t)$. Let $U=U^{1}-U^{2}$ and $U_{0}=U_{0}^{1}-U_{0}^{2}$. Thus, $U$ satisfies

$$
\begin{cases}\rho_{1} u_{t t}-k\left(u_{x}+v\right)_{x}+\gamma \theta_{x}=0, & x \in(0,1), t>0, \\ \rho_{2} v_{t t}-b_{0} v_{x x}+k\left(u_{x}+v\right)-\gamma \theta & \\ \quad-\int_{0}^{+\infty} h(s) \eta_{x x}(x, s) d s+g\left(v^{1}\right)-g\left(v^{2}\right)=0, & x \in(0,1), t>0, \\ \rho_{3} \theta_{t}-\beta \theta_{x x}+\gamma\left(u_{x}+v\right)_{t}=0, & x \in(0,1), t>0, \\ \eta_{t}+\eta_{s}-v_{t}=0, & x \in(0,1), t, s>0\end{cases}
$$

with same boundary conditions as in 2.9. Next, we multiply $4.1{ }_{1}$ by $\bar{u}_{t}, 4.1{ }_{2}$ by $v_{t}$, and 4.1 3 by $\theta$, making use of 4.1 4 , then perform integration by parts over $(0,1)$. Addition of the results from these leads to

$$
\frac{d}{d t} E_{1}(t)=-\beta\left\|\theta_{x}\right\|^{2}-\left(g\left(v^{1}\right)-g\left(v^{2}\right), v_{t}\right)+\frac{1}{2} \int_{0}^{1} \int_{0}^{\infty} h^{\prime}(s)\left|\eta_{x}(x, s)\right|^{2} d s d x,
$$

where

$$
E_{1}(t)=\frac{1}{2}\left(\rho_{1}\left\|\bar{u}_{t}\right\|^{2}+\rho_{2}\left\|v_{t}\right\|^{2}+b_{0}\left\|v_{x}\right\|^{2}+\rho_{3}\|\theta\|^{2}+\left\|\eta_{x}\right\|_{\mathcal{M}}^{2}+k\left\|u_{x}+v\right\|^{2}\right) .
$$

We have

$$
\begin{aligned}
-\left(g\left(v^{1}\right)-g\left(v^{2}\right), v_{t}\right) & \leq \rho_{2}\left(b-b_{0}\right)\left\|v_{t}\right\|^{2}+c \int_{0}^{1}\left|g\left(v^{1}\right)-g\left(v^{2}\right)\right|^{2} d x \\
& \leq \rho_{2}\left(b-b_{0}\right)\left\|v_{t}\right\|^{2}+c\left\|g^{\prime}(y)\right\|_{L^{\infty}(0,1)}^{2} \int_{0}^{1}|v|^{2} d x \\
& \leq \rho_{2}\left(b-b_{0}\right)\left\|v_{t}\right\|^{2}+C_{\mathcal{B}}\|v\|^{2} .
\end{aligned}
$$

Hence from (4.18) and 4.20 , we conclude that

$$
\frac{d}{d t} E_{1}(t) \leq-\beta\left\|\theta_{x}\right\|^{2}+\frac{\rho_{2}\left(b-b_{0}\right)}{2}\left\|v_{t}\right\|^{2}+\frac{1}{2} \int_{0}^{1} \int_{0}^{\infty} h^{\prime}(s)\left|\eta_{x}(x, s)\right|^{2} d s d x+C_{\mathcal{B}}\|v\|^{2}
$$

Let the functional $\mathscr{F}(t)$ be defined as

$$
\mathscr{F}(t)=n E_{1}(t)+n_{1} L_{1}(t)+n_{2} L_{2}(t)+n_{3} L_{3}(t)+L_{4}(t)
$$

where $n, n_{1}, n_{2}$ and $n_{3}$ are positive constants to be chosen later, while

$$
\begin{array}{ll}
L_{1}(t)=-\rho_{3} \int_{0}^{1} \theta \int_{0}^{x} \bar{u}_{t}(y) d y d x, & L_{2}(t)=-\rho_{1} \int_{0}^{1}\left(u_{x}-v\right) \int_{0}^{x} \bar{u}_{t}(y) d y d x, \\
L_{3}(t)=-\rho_{2} \int_{0}^{1} v_{t} \int_{0}^{+\infty} h(s) \eta(x, s) d s d x, & L_{4}(t)=\rho_{2} \int_{0}^{1} v v_{t} d x .
\end{array}
$$


We have that

$$
\varpi_{1} E_{1}(t) \leq \mathscr{F}(t) \leq \varpi_{2} E_{1}(t), \quad \forall t \geq 0
$$

for some positive constants $\varpi_{1}$ and $\varpi_{2}$. Indeed, making use of Young, Poincaré and Cauchy-Schwarz inequalities, we have

$$
\begin{aligned}
& \left|\mathscr{F}(t)-n E_{1}(t)\right| \\
\leq & n_{1}\left|L_{1}(t)\right|+n_{2}\left|L_{2}(t)\right|+n_{3}\left|L_{3}(t)\right|+\left|L_{4}(t)\right| \\
\leq & n_{1} \rho_{3}^{2}\|\theta\|^{2}+c\left(n_{1}+n_{2}\right)\left\|\bar{u}_{t}\right\|+n_{2} \rho_{1}^{2}\left\|u_{x}+v\right\|^{2} \\
& +n_{3} \rho_{2}^{2}\left\|v_{t}\right\|^{2}+c n_{3}\left(b-b_{0}\right) \lambda_{1}\left\|\eta_{x}\right\|_{\mathcal{M}}^{2}+\rho_{2}^{2} \lambda_{1}\left\|v_{x}\right\|^{2}+c\left\|v_{t}\right\|^{2} \\
\leq & c\left(\rho_{1}\left\|\bar{u}_{t}\right\|^{2}+\rho_{2}\left\|v_{t}\right\|^{2}+b_{0}\left\|v_{x}\right\|^{2}+\rho_{3}\|\theta\|^{2}+\left\|\eta_{x}\right\|_{\mathcal{M}}^{2}+k\left\|u_{x}+v\right\|^{2}\right) \\
\leq & c E_{1}(t) .
\end{aligned}
$$

Thus for $n$ large, we deduce 4.22 .

Lemma 4.13. There exists $\varpi>0$ such that

$$
\frac{d}{d t} \mathscr{F}(t)+\varpi \mathscr{F}(t) \leq C_{\mathcal{B}}\|v(t)\|^{2}, \quad \forall t \geq 0 .
$$

Proof. Performing similar computations as those of Lemmas 4.5 4.8, we obtain

$$
\begin{aligned}
& L_{1}^{\prime}(t) \leq-\frac{\gamma}{2}\left\|\bar{u}_{t}\right\|^{2}+\varepsilon_{1}\left\|u_{x}+v\right\|^{2}+c\left(1+\frac{1}{\varepsilon_{1}}\right)\left\|\theta_{x}\right\|^{2}+c\left\|v_{t}\right\|^{2}, \\
& L_{2}^{\prime}(t) \leq-\frac{k}{2}\left\|u_{x}+v\right\|^{2}+c\left\|\theta_{x}\right\|^{2}+c\left\|\bar{u}_{t}\right\|^{2}+c\left\|v_{t}\right\|^{2}, \\
& L_{3}^{\prime}(t) \leq-\frac{\rho_{2}\left(b-b_{0}\right)}{2}\left\|v_{t}\right\|^{2}+\varepsilon_{1}\left\|v_{x}\right\|^{2}+\varepsilon_{2}\left\|u_{x}+v\right\|^{2}+\varepsilon_{2}\left\|\theta_{x}\right\|^{2}+C_{\mathcal{B}}\|v\|^{2} \\
& +c\left(1+\frac{1}{\varepsilon_{2}}+\frac{1}{\varepsilon_{1}}\right)\left\|\eta_{x}\right\|_{\mathcal{M}}^{2}-c \int_{0}^{1} \int_{0}^{+\infty} h^{\prime}(s)\left|\eta_{x}(x, s)\right|^{2} d s d x, \\
& L_{4}^{\prime}(t) \leq-\frac{b_{0}}{2}\left\|v_{x}\right\|^{2}+\rho_{2}\left\|v_{t}\right\|^{2}+c\left\|u_{x}+v\right\|^{2}+c\left\|\theta_{x}\right\|^{2}+C_{\mathcal{B}}\|v\|^{2}+\left(b-b_{0}\right)\left\|\eta_{x}\right\|_{\mathcal{M}}^{2} \text {. }
\end{aligned}
$$

It follows from 4.21 and (4.24) that

$$
\begin{aligned}
\frac{d}{d t} \mathscr{F}(t) \leq & -\left[\frac{n_{1} \gamma}{2}-n_{2} c\right]\left\|\bar{u}_{t}\right\|^{2}-\left[\frac{\rho_{2}\left(b-b_{0}\right)}{4} n_{3}-c n_{1}-c n_{2}-\rho_{2}\right]\left\|v_{t}\right\|^{2} \\
& -\left[n \beta-c\left(1+\frac{1}{\varepsilon_{2}}\right) n_{1}-c n_{2}-\varepsilon_{2} n_{3}+c\right]\left\|\theta_{x}\right\|^{2}-\left[\frac{b_{0}}{2}-\varepsilon_{1} n_{3}\right]\left\|v_{x}\right\|^{2} \\
& -\left[n_{2} \frac{k}{2}-\varepsilon_{2} n_{1}-\varepsilon_{1} n_{3}-c\right]\left\|u_{x}+v\right\|^{2} \\
& +\left[c n_{3}\left(1+\frac{1}{\varepsilon_{2}}+\frac{1}{\varepsilon_{1}}\right)+\left(b-b_{0}\right)\right]\left\|\eta_{x}\right\|_{\mathcal{M}}^{2} \\
& +\left[\frac{n}{2}-c n_{3}\right] \int_{0}^{1} \int_{0}^{+\infty} h^{\prime}(s)\left|\eta_{x}(x, s)\right|^{2} d s d x .
\end{aligned}
$$


Now, with appropriate choices of $\varepsilon_{1}, \varepsilon_{2}, n, n_{1}, n_{2}, n_{3}$ done in a manner similar to that of Lemma 4.16, we obtain

$$
\frac{d}{d t} \mathscr{F}(t) \leq C E_{1}(t)+C_{\mathcal{B}}\|v(t)\|^{2}
$$

Therefore, 4.23 follows from 4.22 and 4.25.

Theorem 4.14. Under the assumptions of Theorem 3.1, the semigroup $S(t)$ of system 2.7) 2.9 is asymptotically smooth in $\mathcal{H}$.

Proof. We integrate (4.23) over $(0, t)$, then on account of 4.22 , we deduce

$$
E_{1}(t) \leq \frac{\varpi_{2}}{\varpi_{1}} E_{1}(0) e^{-\varpi t}+C_{\mathcal{B}} \int_{0}^{t}\|v(s)\|^{2} d s .
$$

Using 4.19, we obtain

$$
\left\|\left(\bar{u}, \bar{u}_{t}, v, v_{t}, \theta, \eta\right)\right\|_{\mathcal{H}}^{2} \leq C\left\|\left(\bar{u}_{0}, \bar{u}_{1}, v_{0}, v_{1}, \theta_{0}, \eta^{0}\right)\right\|_{\mathcal{H}}^{2}+C_{\mathcal{B}} \int_{0}^{t}\|v(s)\|^{2} d s .
$$

Thus

$$
\|U\|_{\mathcal{H}}^{2} \leq C\left\|U_{0}\right\|_{\mathcal{H}}^{2}+C_{\mathcal{B}} \int_{0}^{t}\|v(s)\|^{2} d s .
$$

Let $\epsilon>0$ and select $T$ large enough in 4.26 , we deduce that

$$
\left\|S(T) U_{0}^{1}-S(T) U_{0}^{2}\right\|_{\mathcal{H}}^{2} \leq \epsilon+\Psi_{T}\left(U_{0}^{1}, U_{0}^{2}\right)
$$

for every $U_{0}^{1}, U_{0}^{2} \in \mathcal{B}$, where the function $\Psi_{T}: \mathcal{H} \times \mathcal{H} \rightarrow \mathbb{R}$ is defined by

$$
\Psi_{T}\left(U_{0}^{1}, U_{0}^{2}\right)=C_{\mathcal{B}} \sup _{0 \leq t \leq T} \int_{0}^{t}\left\|v^{1}(s)-v^{2}(s)\right\|^{2} d s .
$$

Let $\left\{\left(\bar{u}_{0 n}, \bar{u}_{1 n}, v_{0 n}, v_{1 n}, \theta_{0 n}, \eta_{0 n}\right)\right\}_{n \geq 1} \subset \mathcal{B}$, since $\mathcal{B} \subset \mathcal{H}$ is bounded and positively invariant, then the corresponding solutions

$$
\left\{\left(\bar{u}_{n}(t),\left(\bar{u}_{t}\right)_{n}(t), v_{n}(t),\left(v_{t}\right)_{n}(t), \theta_{n}(t), \eta_{n}(s)\right)\right\}_{n \geq 1}
$$

is uniformly bounded in $\mathcal{H}$. Hence

$$
\left\{\left(\bar{u}_{n}(t),\left(\bar{u}_{t}\right)_{n}(t), v_{n}(t),\left(v_{t}\right)_{n}(t), \theta_{n}(t), \eta_{n}(s)\right)\right\}_{n \geq 1}
$$

is a bounded sequence in

$$
C([0, T], \mathcal{H})
$$

It follows that $\left\{v_{n}(t)\right\}$ is bounded in

$$
C\left([0, T], H_{0}^{1}(0,1)\right) .
$$


Using the compact embedding of $C\left([0, T], H_{0}^{1}(0,1)\right) \cap C^{1}\left([0, T], L^{2}(0,1)\right)$ into $C([0, T]$, $\left.L^{2}(0,1)\right)$, we can extract a subsequence $\left\{v_{n_{j}}(t)\right\}$ which converges strongly in

$$
C\left([0, T], L^{2}(0,1)\right), \quad \forall T>0 .
$$

Therefore

$$
\lim _{j \rightarrow \infty} \lim _{l \rightarrow \infty} \sup _{0 \leq t \leq T} \int_{0}^{t}\left\|v_{n_{j}}(s)-v_{n_{l}}(s)\right\|^{2} d s=0
$$

and so

$$
\lim _{j \rightarrow \infty} \lim _{l \rightarrow \infty} \Psi_{T}\left(U_{0 n_{j}}, U_{0 n_{l}}\right)=0 .
$$

By applying Lemma 4.12, we obtain the asymptotic smoothness of the semigroup $S(t)$ in $\mathcal{H}$.

Theorem 4.15. Under the assumptions of Theorem 3.1, the semigroup $S(t)$ of system 2.7)-2.9) possesses the compact global attractor $\mathcal{A}$ in $\mathcal{H}$.

Proof. In Theorem 4.11, we showed that the semigroup $S(t)$ of system 2.7 -2.9 possesses a bounded absorbing set $\mathscr{B}$ in $\mathcal{H}$, and furthermore showed in Theorem 4.14 that the semigroup $S(t)$ of system $2.7-2.9$ is asymptotically smooth in $\mathcal{H}$. The result follows by the application of Theorem 4.4 .

\section{Finite-fractal dimension}

We devote this section, to showing that the global attractor $\mathcal{A}$ obtained in Section 4 possesses a finite-fractal dimension. We first present some needed basic concepts and results. The reader is referred to $[5]$ and references therein for more details.

Let $X$ be a metric space and $K \subset X$ be a compact set, then the fractal dimension of $K$ is given by

$$
\operatorname{dim}_{f}^{X} K=\limsup _{\epsilon \rightarrow 0} \frac{\operatorname{In}(n(K, \epsilon))}{\operatorname{In}(1 / \epsilon)},
$$

where $n(K, \epsilon)$ is the minimal number of closed balls with radius $\epsilon$ that cover $K$.

Suppose $n_{X}(\cdot)$ is a seminorm on a Banach space $X$, it is known that $n_{X}$ is compact whenever for any sequence $x_{j} \rightarrow 0$ weakly in $X$ we have that $n_{X}\left(x_{j}\right) \rightarrow 0$.

Let $X, V, Z$ be three reflexive Banach spaces with $X$ compactly embedded in $V$, we set $H=X \times V \times Z$. We consider the dynamical system $(H, S(t))$ where

$$
S(t)\left(y_{0}, y_{1}, z_{0}\right)=\left(y(t), y_{t}(t), z(t)\right), \quad\left(y_{0}, y_{1}, z_{0}\right) \in H
$$

is the solution of an evolution problem, and $y, z$ satisfies the regularity

$$
y \in \mathcal{C}([0,+\infty), X) \cap \mathcal{C}^{1}([0,+\infty), V), \quad z \in \mathcal{C}([0,+\infty), Z) .
$$

We state the following important definition and theorem. 
Definition 5.1. The dynamical system $(H, S(t))$ is said to be quasi-stable on a set $\mathcal{U} \subset H$ if there exists a compact seminorm $n_{X}$ on $X$ and nonnegative scalar functions $\alpha(t)$ and $\phi(t)$ which are locally bounded in $[0,+\infty)$, and $\beta(t) \in L^{1}((0,+\infty))$ with $\lim _{t \rightarrow+\infty} \beta(t)=0$, such that

$$
\left\|S(t)\left(y_{0}^{1}, y_{1}^{1}, z_{0}^{1}\right)-S(t)\left(y_{0}^{2}, y_{1}^{2}, z_{0}^{2}\right)\right\|_{H}^{2} \leq \alpha(t)\left\|\left(y_{0}^{1}, y_{1}^{1}, z_{0}^{1}\right)-\left(y_{0}^{2}, y_{1}^{2}, z_{0}^{2}\right)\right\|_{H}^{2},
$$

and

$$
\begin{aligned}
\left\|S(t)\left(y_{0}^{1}, y_{1}^{1}, z_{0}^{1}\right)-S(t)\left(y_{0}^{2}, y_{1}^{2}, z_{0}^{2}\right)\right\|_{H}^{2} \leq & \beta(t)\left\|\left(y_{0}^{1}, y_{1}^{1}, z_{0}^{1}\right)-\left(y_{0}^{2}, y_{1}^{2}, z_{0}^{2}\right)\right\|_{H}^{2} \\
& +\phi(t) \sup _{0<s<t}\left[n_{X}\left(y^{1}(s)-y^{2}(s)\right)\right]^{2}
\end{aligned}
$$

for any $\left(y_{0}^{1}, y_{1}^{1}, z_{0}^{1}\right),\left(y_{0}^{2}, y_{1}^{2}, z_{0}^{2}\right) \in \mathcal{U}$. The inequality (5.4) is known as a stabilizability inequality.

Theorem 5.2. [5] Let the dynamical system $(H, S(t))$ be given by (5.1) and satisfy (5.2). If $(H, S(t))$ possesses a compact global attractor $\mathcal{A}$ and is quasi-stable on $\mathcal{A}$, then the global attractor $\mathcal{A}$ has finite fractal dimension.

Here is the main result of this section.

Theorem 5.3. The global attractor obtained in Theorem 4.15 possesses a finite-fractal dimension.

Proof. Let $X=H_{*}^{1}(0,1) \times H_{0}^{1}(0,1), V=L^{2}(0,1) \times L^{2}(0,1)$ and $Y=L^{2}(0,1) \times \mathcal{M}$. Also, set $y(t)=(\bar{u}, v), y_{t}(t)=\left(\bar{u}_{t}, v_{t}\right), z(t)=(\theta, \eta)$. Therefore, the dynamical system $(\mathcal{H}, S(t))$ emanating from the solution of system (2.7)-(2.9) satisfies (5.1) and (5.2). Let $\mathfrak{B} \subset \mathcal{H}$, be a bounded positively invariant set with respect to $S(t)$. Let

$$
U_{0}^{j}=\left(\bar{u}_{0}^{i}, \bar{u}_{1}^{i}, v_{0}^{i}, v_{1}^{i}, \theta_{0}^{i}, \eta^{0 i}\right), \quad i=1,2
$$

We can see that $U=U^{1}-U^{2}$ satisfies 4.1). Hence, with similar computations as in Lemma 4.1, we simply obtain

$$
\left\|S(t) U_{0}^{1}-S(t) U_{0}^{2}\right\|_{\mathcal{H}}^{2} \leq e^{\vartheta t}\left\|U_{0}^{1}-U_{0}^{2}\right\|_{\mathcal{H}}^{2}, \quad \forall t \geq 0
$$

for any $U_{0}^{1}, U_{0}^{2} \in \mathfrak{B}$, where $\vartheta=\vartheta(\mathfrak{B})>0$. Hence, we set $\alpha(t)=e^{\vartheta t}$ in 5.3 and clearly, $\alpha(t)$ is locally bounded in $[0,+\infty)$. We now show that $(5.4)$ is satisfied. Let's define the seminorm $n_{X}(\cdot)$ by

$$
n_{X}(u, v)=\|u\|+\|v\|
$$


We know that $H_{0}^{1}(0,1)$ is compactly embedded in $L^{2}(0,1)$. Hence, the seminorm $n_{X}(\cdot)$ is compact on $H_{0}^{1}(0,1)$. We integrate 4.23 over $(0, t)$, then on account of 4.22 , we obtain

$$
\begin{aligned}
\left\|S(t) U_{0}^{1}-S(t) U_{0}^{2}\right\|_{\mathcal{H}}^{2} & \leq C e^{-\varpi t}\left\|U_{0}^{1}-U_{0}^{2}\right\|_{\mathcal{H}}^{2}+C_{\mathfrak{B}} \int_{0}^{t} e^{-\varpi(t-s)}\left\|v^{1}-v^{2}\right\|^{2} d s \\
& \leq C e^{-\varpi t}\left\|U_{0}^{1}-U_{0}^{2}\right\|_{\mathcal{H}}^{2}+C_{\mathfrak{B}} \int_{0}^{t} e^{-\varpi(t-s)} d s \sup _{0<s<t}\left[n_{X}(y)\right]^{2} \\
& =\beta(t)\left\|U_{0}^{1}-U_{0}^{2}\right\|_{\mathcal{H}}^{2}+\phi(t) \sup _{0<s<t}\left[n_{X}(y)\right]^{2},
\end{aligned}
$$

where

$$
y=\left(u^{1}-u^{2}, v^{1}-v^{2}\right), \quad \beta(t)=C e^{-\varpi t}, \quad \phi(t)=C_{\mathfrak{B}} \int_{0}^{t} e^{-\varpi(t-s)} d s, \quad t \geq 0 .
$$

We easily see that

$$
\beta(t) \in L^{1}\left(\mathbb{R}^{+}\right) \quad \text { and } \quad \lim _{t \rightarrow 0} \beta(t)=0 .
$$

The boundedness of $\mathfrak{B}$ implies that $\phi(t)$ is locally bounded in $[0, \infty)$. Hence, the condition (5.4) is satisfied. Therefore, the dynamical system $(\mathcal{H}, S(t))$ is quasi-stable on $\mathcal{A}$. Application of Theorem 5.2 , gives that the global attractor $\mathcal{A}$ has a finite-fractal dimension.

\section{Exponential attractor}

This section is devoted to show that the system (2.7)-2.9 possesses an exponential attractor.

Definition 6.1 (Exponential attractor). Let $X$ be a compact subset of $\mathcal{H}$. A compact set $\mathcal{E} \subset X$ is called an exponential attractor for the semigroup $S(t)$ for the topology of $\mathcal{H}$ if

(i) $\mathcal{E}$ is positively invariant under $S(t)$, that is, $S(t) \mathcal{E} \subset \mathcal{E}, \forall t \geq 0$;

(ii) the fractal dimension of $\mathcal{E}$ is finite;

(iii) there exists a constant $c_{0}>0$ such that, for every bounded subset $B \subset X$, there exists a constant $c_{1}(B)>0$ such that

$$
\operatorname{dist}_{\mathcal{H}}(S(t) B, \mathcal{E}) \leq c_{1} e^{-c_{0} t}, \quad \forall t \geq 0 .
$$

Furthermore, we introduce the concept of generalized exponential attractors, in which case the exponential attractor is said to have fractal dimension in a weaker phase space $\widetilde{\mathcal{H}} \supseteq \mathcal{H}$, see $[5,6$ for more details.

We denote by $H^{-1}(0,1)$ the dual of $H_{0}^{1}(0,1)$, and set

$$
\widetilde{\mathcal{H}}=L^{2}(0,1) \times H^{-1}(0,1) \times L^{2}(0,1) \times H^{-1}(0,1) \times H^{-1}(0,1) \times \mathcal{M}^{\prime},
$$

where $\mathcal{M}^{\prime}=L^{2}\left(\mathbb{R}^{+}, L^{2}(0,1)\right)$. We state the following result of Chueshov and Lasiecka 6 . 
Theorem 6.2. Let $(\mathcal{H}, S(t))$ be a dynamical system satisfying (5.1) and (5.2). Assume $(\mathcal{H}, S(t))$ is quasi-stable on a bounded absorbing set $\mathscr{B}$, and there exists an extended space $\widetilde{\mathcal{H}} \supseteq \mathcal{H}$, such that for any $T^{*}>0$,

$$
\left\|S(t) U_{0}-S\left(t^{\prime}\right) U_{0}\right\|_{\widetilde{\mathcal{H}}} \leq C^{*}\left|t-t^{\prime}\right|^{\sigma}, \quad t, t^{\prime} \in\left[0, T^{*}\right], U_{0} \in \mathscr{B}
$$

where the constants $C^{*}=C^{*}\left(\mathscr{B}, T^{*}\right)>0$ and $0<\sigma \leq 1$. Then $(\mathcal{H}, S(t))$ possesses a generalized exponential attractor $\mathcal{E} \subset \mathcal{H}$ with finite fractal dimension in $\widetilde{\mathcal{H}}$.

The next theorem is the main result of this section.

Theorem 6.3. Under the assumptions of Theorem 3.1, the semigroup $S(t)$ of system (2.7)2.9 possesses a generalized exponential attractor $\mathcal{E}$ in $\mathcal{H}$, with finite fractal dimension in the extended space $\widetilde{\mathcal{H}}$.

Proof. Let $\mathscr{B} \subset \mathcal{H}$, be a bounded positively invariant set with respect to $S(t)$. Similar to the proof in Theorem 5.3 , the dynamical system $(\mathcal{H}, S(t))$ is quasi-stable on $\mathscr{B}$.

Let $U_{0} \in \mathscr{B}$, then differentiating the system $\left.(2.7)-2.9\right)$, we deduce that the solution $\left(\bar{u}, \bar{u}_{t}, v, v_{t}, \theta, \eta\right)$ satisfies

$$
\rho_{1}\left\|u_{t t}\right\|_{-1}^{2}+\rho_{2}\left\|v_{t t}\right\|_{-1}^{2}+\rho_{3}\left\|\theta_{t}\right\|_{-1}^{2}+b_{0}\left\|v_{x t}\right\|_{-1}^{2}+k\left\|u_{x t}+v_{t}\right\|_{-1}^{2}+\left\|\eta_{t}\right\|_{\mathcal{M}^{\prime}}^{2} \leq C_{\mathscr{B}} .
$$

We have that

$$
\begin{aligned}
& \left\|S(t) U_{0}-S\left(t^{\prime}\right) U_{0}\right\|_{\widetilde{\mathcal{H}}} \\
\leq & \rho_{1}\left\|u_{t}(t)-u_{t}\left(t^{\prime}\right)\right\|_{-1}+\rho_{2}\left\|v_{t}(t)-v_{t}\left(t^{\prime}\right)\right\|_{-1}+\rho_{3}\left\|\theta(t)-\theta\left(t^{\prime}\right)\right\|_{-1} \\
& +b_{0}\left\|v_{x}(t)-v_{x}\left(t^{\prime}\right)\right\|_{-1}+k\left\|u_{x}(t)-u_{x}\left(t^{\prime}\right)+v(t)-v\left(t^{\prime}\right)\right\|_{-1}+\left\|\eta(t)-\eta\left(t^{\prime}\right)\right\|_{\mathcal{M}^{\prime}} \\
\leq & \int_{t}^{t^{\prime}}\left(\left\|u_{t t}(s)\right\|_{-1}+\left\|v_{t t}(s)\right\|_{-1}+\left\|\theta_{t}(s)\right\|_{-1}+\left\|v_{x t}(s)\right\|_{-1}\right) d s \\
& +\int_{t}^{t^{\prime}}\left(\left\|u_{x t}(s)+v_{t}(s)\right\|_{-1}+\left\|\eta_{t}(s)\right\|_{\mathcal{M}^{\prime}}\right) d s .
\end{aligned}
$$

It follows from $(6.2)$ and $(6.3)$ that

$$
\left\|S(t) U_{0}-S\left(t^{\prime}\right) U_{0}\right\|_{\widetilde{\mathcal{H}}} \leq C^{*}\left(\mathscr{B}, T^{*}\right)\left|t-t^{\prime}\right|^{1 / 2}, \quad t, t^{\prime} \in\left[0, T^{*}\right]
$$

Therefore (6.1) holds, and we conclude that $(\mathcal{H}, S(t))$ has a generalized exponential attractor with finite fractal dimension in $\widetilde{\mathcal{H}}$.

\section{Acknowledgments}

The authors appreciate the continuous support of the University of Hafr Al Batin. 


\section{References}

[1] F. Ammar-Khodja, A. Benabdallah, J. E. Muñoz Rivera and R. Racke, Energy decay for Timoshenko systems of memory type, J. Differential Equations 194 (2003), no. 1, $82-115$.

[2] T. A. Apalara, Asymptotic behavior of weakly dissipative Timoshenko system with internal constant delay feedbacks, Appl. Anal. 95 (2016), no. 1, 187-202.

[3] _ General stability of memory-type thermoelastic Timoshenko beam acting on shear force, Contin. Mech. Thermodyn. 30 (2018), no. 2, 291-300.

[4] M. M. Cavalcanti, V. N. Domingos Cavalcanti, F. A. Falcão Nascimento, I. Lasiecka and J. H. Rodrigues, Uniform decay rates for the energy of Timoshenko system with the arbitrary speeds of propagation and localized nonlinear damping, Z. Angew. Math. Phys. 65 (2014), no. 6, 1189-1206.

[5] I. Chueshov and I. Lasiecka, Long-time behavior of second order evolution equations with nonlinear damping, Mem. Amer. Math. Soc. 195 (2008), no. 912, 183 pp.

[6] _ Von Karman Evolution Equations: Well-posedness and long-time dynamics, Springer Monographs in Mathematics, Springer, New York, 2010.

[7] F. Dell'Oro and V. Pata, On the stability of Timoshenko systems with Gurtin-Pipkin thermal law, J. Differential Equations 257 (2014), no. 2, 523-548.

[8] L. H. Fatori, M. A. Jorge Silva and V. Narciso, Quasi-stability property and attractors for a semilinear Timoshenko system, Discrete Contin. Dyn. Syst. 36 (2016), no. 11, 6117-6132.

[9] B. Feng and X.-G. Yang, Long-time dynamics for a nonlinear Timoshenko system with delay, Appl. Anal. 96 (2017), no. 4, 606-625.

[10] A. Guesmia and S. A. Messaoudi, On the control of a viscoelastic damped Timoshenkotype system, Appl. Math. Comput. 206 (2008), no. 2, 589-597.

[11] General energy decay estimates of Timoshenko systems with frictional versus viscoelastic damping, Math. Methods Appl. Sci. 32 (2009), no. 16, 2102-2122.

[12] A. Guesmia, S. A. Messaoudi and A. Soufyane, Stabilization of a linear Timoshenko system with infinite history and applications to the Timoshenko-heat systems, Electron. J. Differential Equations 2012 (2012), no. 193, 45 pp. 
[13] S. M. Han, H. Benaroya and T. Wei, Dynamics of transversely vibrating beams using four engineering theories, J. Sound Vibration 225 (1999), no. 5, 935-988.

[14] J. U. Kim and Y. Renardy, Boundary control of the Timoshenko beam, SIAM J. Control Optim. 25 (1987), no. 6, 1417-1429.

[15] A. Lo and N.-e. Tatar, Stabilization of laminated beams with interfacial slip, Electron. J. Differential Equations 2015 (2015), no. 129, 14 pp.

[16] S. A. Messaoudi and A. Fareh, General decay for a porous thermoelastic system with memory: the case of equal speeds, Nonlinear Anal. 74 (2011), no. 18, 6895-6906.

[17] _ General decay for a porous-thermoelastic system with memory: the case of nonequal speeds, Acta Math. Sci. Ser. B (Engl. Ed.) 33 (2013), no. 1, 23-40.

[18] S. A. Messaoudi and M. I. Mustafa, A stability result in a memory-type Timoshenko system, Dynam. Systems Appl. 18 (2009), no. 3-4, 457-468.

[19] S. A. Messaoudi and B. Said-Houari, Uniform decay in a Timoshenko-type system with past history, J. Math. Anal. Appl. 360 (2009), no. 2, 459-475.

[20] Energy decay in a Timoshenko-type system with history in thermoelasticity of type III, Adv. Differential Equations 14 (2009), no. 3-4, 375-400.

[21] N.-E. Tatar, Stabilization of a laminated beam with interfacial slip by boundary controls, Bound. Value Probl. 2015 (2015), no. 169, 11 pp.

Soh Edwin Mukiawa and Cyril Dennis Enyi

Department of Mathematics and Statistics, University of Hafr Al Batin, Hafar Al Batin 39524, Saudi Arabia

E-mail address: mukiawa@uhb.edu.sa, cyrild@uhb.edu.sa 